\title{
Deterioration of Pre-Existing Hemiparesis Brought About by Subsequent Ipsilateral Infarction-A Case Report
}

\author{
Kewei Yu, Gang Liu, Junfa Wu, Ying Chen and Yi Wu* \\ Department of Rehabilitation Medicine, Huashan Hospital, China
}

*Corresponding author: Yi Wu, Department of Rehabilitation Medicine, Huashan Hospital, Shanghai 200040, China

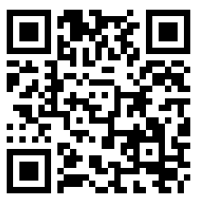

\section{ARTICLE INFO}

Received: 粜 September 03, 2019

Published: 㓞 September 09, 2019

Citation: Kewei Yu, Gang Liu, Junfa Wu, Ying Chen, Yi Wu. Deterioration of Pre-Existing Hemiparesis Brought About by Subsequent Ipsilateral Infarction-A Case Report. Biomed J Sci \& Tech Res 21(2)-2019. BJSTR. MS.ID.003562.

Keywords: Stroke; Compensation

\begin{abstract}
Reorganization of the central neural system is the main mechanism of post-stroke recovery. Several evidences suggest that ipsilateral uncrossed corticospinal tracts play an important role in the recovery of motor function after stroke. A 68-year-old woman has right-sided hemiparesis because of her left basal ganglia cerebral infarction. However, she presented with abrupt deterioration of her right-sided hemiparesis due to subsequent infarcts in the right basal ganglia cerebral infarction half year later. Therefore, we speculate the subsequent right basal ganglia cerebral infarction may have damaged the uncrossed tract, thereby causing the pre-existing hemiparesis to deteriorate even further. This suggests that the uncrossed corticospinal tracts participate in the recovery of the right-sided hemiparesis after stroke. Hemiplegic limb motor function in stroke patients would get better after rehabilitation therapy. However, the mechanism of this phenomenon is still poorly understood. We report a case where cortical reorganization of the unaffected hemisphere may have played a significant role in motor function in stroke patients, but where this partial recovery of hemiparesis appears to have been damaged by a subsequent ipsilateral basal ganglia cerebral infarction half year later.
\end{abstract}

Case Report
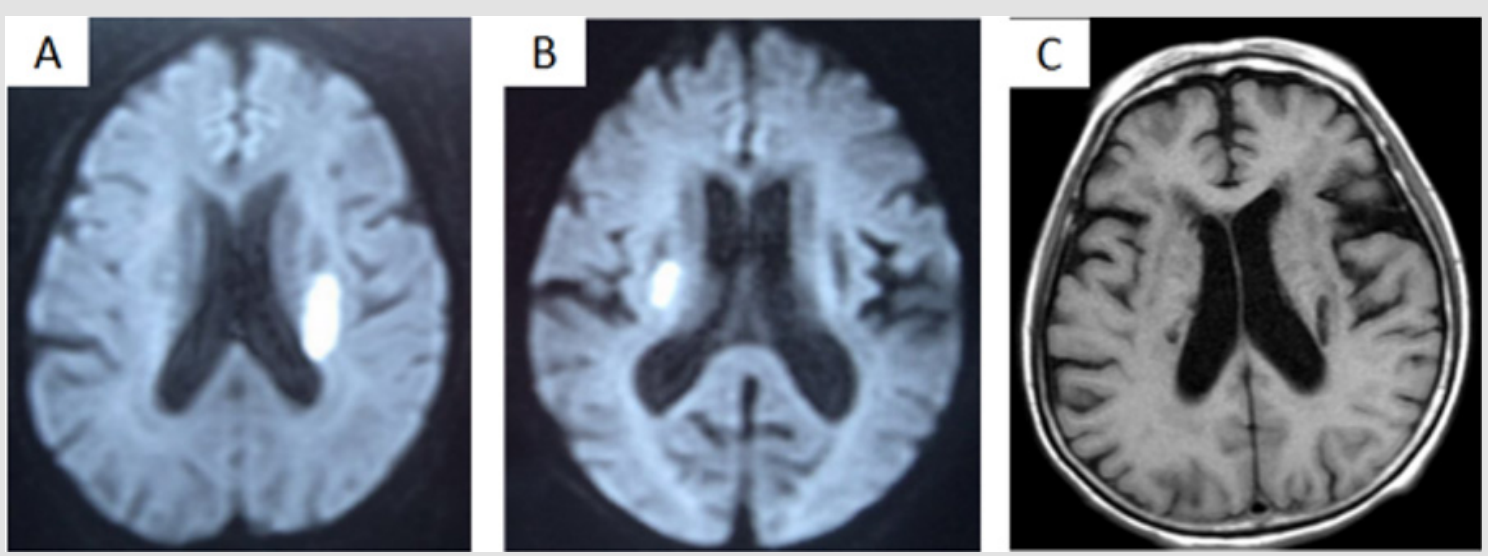

Figure 1:

a) Images indicating previous stroke.

b) Images indicating secondary strokes.

c) The bilateral lateral cerebral infarction lesions one year later. 
A 68-year-old woman was admitted to our hospital because limbs activity is not flexible and dysphagia, especially for the deterioration in pre-existing right hemiparesis. The patient had a history of left basal ganglia cerebral infarction (Figure $1 \mathrm{~A}$ ) half year earlier, and she had a mild right hemiparesis after about six months of rehabilitation therapy. Neurological examination showed that the preexisting left hemiparesis had deteriorated, and the function of her limbs on the left side was moderately affected. Cranial magnetic resonance diffusion weighted imaging(DWI) showed a small high intensity area in the left basal ganglia, which was shown to be cerebral infarction (Figure 1A). However, a new lesion on the right side of the basal ganglia was diagnosed as a infarct by DWI half year later (Figure 1B). Old cerebral infarction lesions were shown in the bilateral lateral ventricle side by magnetic resonance image one year later (Figure 1C).

\section{Discussion}

When an initial stroke results in extensive damage to motor function, a subsequent stroke contralateral to the first stroke sometimes causes deterioration in the ipsilateral pre-existing hemiparesis, as well as producing contralateral motor dysfunction. Some cases involving the deterioration of preexisting hemiparesis brought about by a subsequent ipsilateral corona radiata infarction were also reported, and were similar to our case [1,2]. This mechanism involved is thought to be functional reorganization of the ipsilateral hemisphere. Some studies suggested that latent ipsilateral motor projections are activated by disruption of the contralateral corticospinal projections in stroke patients $[3,4]$. In addition, functional recovery due to the concentration of dopamine increasing in the hemisphere contralateral in the site of stroke [5]. A series of studies have shown that hemisphere contralateral to the lesion site contributes greatly to the functional recovery after a focal stroke of the somatosensory cortex [6-8]. However, most studies on ipsilateral motor projections have reported negative results for motor function $[9,10]$. It is said that enhancement of the ipsilateral motor projections to the paretic side might contribute to generation of an abnormal motor pattern leading to poor motor ability after stroke [11]. Therefore, some studies have shown that peripheral regions of brain injury would play a role in functional compensation $[12,13]$. The contralateral hemispheric activation would facilitate motor recovery [14].

This case report showed that the motor function recovery was due to the hemisphere cortex compensation of the ipsilateral. Therefore, it is hard to decide for us to enhance function compensate whether by increasing the ipsilateral or contralateral cortex after stroke. The current theory shows that stroke patients display an interhemisphericim balance where the contralesional cortex no longer inhibits the ipsilesional hemisphere cortex and the ipsilesional side appears to inhibit the contralesional [15], and lead to functional decline. Therefore, for the transcranial magnetic stimulation treatment, we usually inhibit the ipsilesional cortex and excite contralateral cortex. Mohapatra found that inhibitory TMS of the ipsilesional M1 significantly increased the motor function for stroke patience [16]. In this case, the cortical reorganization of the unaffected hemisphere may have played a significant role in motor function recovery. However, if we inhibit the ipsilesional hemisphere cortex then what would happen to the functional recovery. We Speculate the function might be relatively poor. How should we deal with such patients? We may not always apply this theory: inhibit the ipsilateral cortex and excite contralateral cortex. Therefore, diffusion tensor imaging (DTI) could provide a powerful vehicle for investigating motor recovery mechanisms. Diffusion tensor imaging can evaluate the degree of fiber damage in stroke affecting the corticospinal tracts [17]. If the degree of fiber were damaged seriously in contralateral cortex, we can excite the ipsilateral cortex and strengthen the role of compensatory in the ipsilateral cortex, like this case. Otherwise, we can inhibit the ipsilateral cortex and excite contralateral cortex.

\section{Funding}

The Key Construction Projects of Shanghai Health and Family Planning on Weak Discipline (nos. 2015ZB0401). The key projects of Shanghai Science and Technology on Biomedicine (nos. 17411953900)

\section{Conflict of Interest Declaration}

The authors declare no conflict of interest.

\section{References}

1. Song YM, Lee JY, Park JM (2005) Ipsilateral hemiparesis caused by a corona radiata infarct after a previous stroke on the opposite side. Arch Neurol 62(5): 809-811.

2. Ago T, Kitazono T, Ooboshi H (2003) Deterioration of pre-existing hemiparesis brought about by subsequent ipsilateral lacunar infarction. J Neurol Neurosurg Psychiatry 74(8): 1152-1153.

3. Netz J, Lammers T, Homberg V (1997) Reorganization of motor output in the non-affected hemisphere after stroke. Brain 120 (9): 1579-1586.

4. Werhahn KJ, Conforto AB, Kadom N (2003) Contribution of the ipsilateral motor cortex to recovery after chronic stroke. Ann Neurol 54(4): 464472.

5. Obi K, Amano I, Takatsuru Y (2017) Role of dopamine on functional recovery in the contralateral hemisphere after focal stroke in the somatosensory cortex. Brain Res 1678: 146-152.

6. Takatsuru Y, Fukumoto D, Yoshitomo M (2009) Neuronal circuit remodeling in the contralateral cortical hemisphere during functional recovery from cerebral infarction. J Neurosci 29(32): 10081-10086.

7. Takatsuru Y, Eto K, Kaneko R (2013) Critical role of the astrocyte for functional remodeling in contralateral hemisphere of somatosensory cortex after stroke. J Neurosci 33(11): 4683-4692.

8. Takatsuru Y, Nabekura J, Koibuchi N (2014) Contribution of neuronal and glial circuit in intact hemisphere for functional remodeling after focal ischemia. Neurosci Res 78: 38-44.

9. Netz J, Lammers T, Homberg V (1997) Reorganization of motor output in the non-affected hemisphere after stroke. Brain 120 (9): 1579-1586. 
10. Werhahn KJ, Conforto AB, Kadom N (2003) Contribution of the ipsilateral motor cortex to recovery after chronic stroke. Ann Neurol 54(4): 464472.

11. Takeuchi N, Izumi S (2012) Maladaptive plasticity for motor recovery after stroke: mechanisms and approaches. Neural Plast 2012: 359728.

12. Nudo RJ, Milliken GW (1996) Reorganization of movement representations in primary motor cortex following focal ischemic infarcts in adult squirrel monkeys. J Neurophysiol 75(5): 2144-2149.

13. Morris DM, Taub E (2001) Constraint-induced therapy approach to restoring function after neurological injury. Top Stroke Rehabil 8(3): 1630 .

14. Schaechter JD, Perdue KL (2008) Enhanced cortical activation in the contralesional hemisphere of chronic stroke patients in response to motor skill challenge. Cereb Cortex 18(3): 638-647.

\section{ISSN: 2574-1241}

DOI: 10.26717/BJSTR.2019.21.003562

Yi Wu. Biomed J Sci \& Tech Res

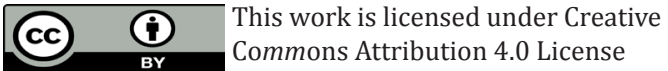

Submission Link: https://biomedres.us/submit-manuscript.php
15. Baron JC, Cohen LG, Cramer SC (2004) Neuroimaging in stroke recovery: a position paper from the First International Workshop on Neuroimaging and Stroke Recovery. Cerebrovasc Dis 18(3): 260-267.

16. Mohapatra S, Harrington R, Chan E (2016) Role of contralesional hemisphere in paretic arm reaching in patients with severe arm paresis due to stroke: A preliminary report. Neurosci Lett 617: 52-58.

17. Beppu T, Nishimoto H, Ishigaki D (2010) Assessment of damage to cerebral white matter fiber in the subacute phase after carbon monoxide poisoning using fractional anisotropy in diffusion tensor imaging. Neuroradiology 52(8): 735-743.

\begin{tabular}{ll} 
BIOMEDICAL & Assets of Publishing with us \\
RESEARCHES & - Global archiving of articles \\
& - Immediate, unrestricted online access \\
\hline
\end{tabular}

